\title{
Allergic rhinitis severity can be assessed using a visual analogue scale in mild, moderate and severe*
}

\author{
Alfonso Del Cuvillo', Vanessa Santos², Javier Montoro ${ }^{3}$, Joan Bartra ${ }^{4,10}$, \\ Ignacio Dávila ${ }^{5}$, Marta Ferrer ${ }^{6}$, Ignacio Jáureguii , Joaquín Sastre ${ }^{8}$, \\ Joaquim Mullol $\left.\right|^{9,10,5}$, Antonio Valero ${ }^{4,10,5}$ \\ 'Unidad de Rinología y asma, UGC ORL Hospital de Jerez del Servicio Andaluz de Salud, Cádiz, Spain
² Fundación para la gestión de la Investigación Biomédica de Cádiz, Spain
${ }^{3}$ Unidad de Alergia, Hospital Universitario Arnau de Vilanova, Facultad de Medicina, Universidad Católica de Valencia "San Vicente \\ Mártir", Valencia, Spain \\ ${ }^{4}$ Unitat d'Al.lèrgia. Servei de Pneumologia i Al.lèrgia Respiratòria, Hospital Clínic, Universitat de Barcelona, CIBERES, Barcelona, Spain \\ ${ }^{5}$ Servicio de Alergia, Hospital Universitario, IBSAL, Salamanca, Spain \\ ${ }^{6}$ Departamento de Alergología e Inmunología Clínica, Clínica Universidad de Navarra, Pamplona, Spain \\ ${ }^{7}$ Servicio de Alergia, Hospital Universitario de Basurto, Bilbao, Spain \\ ${ }^{8}$ Servicio de Alergia, Fundación Jiménez Díaz, CIBERES, Madrid, Spain \\ ${ }^{9}$ Unitat de Rinologia i Clinica de I'Olfacte, Servei d'Otorinolaringologia, Hospital Clínic, Universitat de Barcelona, CIBERES, \\ Barcelona, Spain \\ ${ }^{10}$ Immunoal•lèrgia Respiratòria Clínica i Experimental (IRCE), Institut d'Investigacions Biomèdiques August Pi i Sunyer (IDIBAPS), \\ Research Team of Excellence (2014-SGR-758, Generalitat de Catalunya), Barcelona, Spain
}

Rhinology 55: 34-38, 2017

https://doi.org/10.4193/Rhino16.025

*Received for publication:

January 21, 2016

Accepted: August 8, 2016

${ }^{\$}$ Both authors share similar senior responsibilities

\begin{abstract}
Background: Allergic rhinitis is a global healthcare problem due to its high prevalence, impact on individuals and socioeconomic burden for the nations. Allergic rhinitis severity evaluation is the key to a correct treatment, prevention of comorbidities and improving the quality of life of patients. This evaluation should be made with a simple, easy, fast but accurate and reliable methodology, both in a primary care and specialist setting. The visual analogue scale (VAS) meets all requirements to be the ideal tool to assess allergic rhinitis severity and has already been validated by using a single cut-off point, but this classification in two degrees of severity suffer from not allocating the patients uniformly and from giving a blind interval to classify the patients when the score is between 5 to $6 \mathrm{~cm}$.
\end{abstract}

Methodology: The main objective of our study is to describe the optimal cut-off points by using a VAS to discriminate between three degrees of allergic rhinitis severity (mild, moderate, and severe) following the ARIA modified severity criteria that has been previously validated. Sensitivity, specificity, positive and negative predictive values just like receiver operating characteristic curves were used to select the best cut-off values.

Results: In a cross-sectional multicentre study with 3,572 patients included we have found that VAS has a significant correlation with nasal symptom score and quality of life and that the best cut-off points to differentiate between mild, moderate an severe allergic rhinitis are a VAS score of 4 and 7, respectively.

Conclusions: Allergic rhinitis severity could be assessed in three degrees by using VAS in a simple, easy, and accurate method.

Key words: Allergic rhinitis, severity, visual analogue scale, ARIA 


\section{Introduction}

Allergic rhinitis (AR) is an increasingly prevalent worldwide disease. Although the disease is not life threatening, its severity has been related to an important impact in patients' quality of life, presence of comorbidities (especially asthma) and implies direct and indirect costs due to health care resource consumption, as well as an impairment of work or school performance (1). Given that primary care physicians attend the majority of patients with respiratory diseases, the assessment of severity in primary care should be simple, fast and easy to carry out. It must be validated in order to select those patients who would benefit most from referral to the specialist ${ }^{(2)}$.

AR severity has been assessed using different methods: (i) symptom scales like Total Four Symptom Score (T4SS), that only take symptoms into account, (ii) quality of life scores, that are usually long and difficult to complete in clinical practice, and (iii) the visual analogue scale (VAS), a simple, fast and useful way of evaluating overall AR severity. A previous study has shown the need to better define the cut-off level between categories of severity ${ }^{(3)}$.

In the European Academy of Allergy and Clinical Immunology AR guidelines (Allergic rhinitis and its impact on asthma -ARIA-) the authors have proposed a simple and qualitative severity assessment for AR based on four quality of life items (sleep disturbance, impairment of daily activities, leisure and/or sports, impairment of school or work, troublesome symptoms), distinguishing two categories: mild (patients with none of the items affected) or moderate/severe (patients with one or more items affected). There has been shown that the moderate/severe group is very heterogeneous and includes patients with a wide degree of severity, not discriminating well between them, so that our group has validated a modified criterion to classify AR severity in three degrees in adults and children using the same four ARIA items: mild (no item affected), moderate (one to three items affected), and severe (all four items affected) ${ }^{(4-6)}$. We have also reported that specialist based treatment improves disease severity ${ }^{(7)}$, which finding may help clinicians to classify and follow up AR patients into a more accurately way.

We now present the results of the reanalysis of the database of a previously reported AR epidemiological study in Spain (8) (validating the ARIA classification of AR severity) with the objective of assessing the utility of the VAS to differentiate the degrees of severity of AR.

\section{Materials and methods}

Study design

A multicentre cross sectional study was made, in which 760 investigators from allergy, otorhinolaryngology and general practice centres in Spain, recruited AR patients between March and May 2006.

\section{Study population}

Patients aged 18 years or older with an established diagnosis of AR (positive skin prick tests or specific serum IgE) were consecutively included in the study. All patients signed a written informed consent and the Ethics Committee of Hospital Clínic of Barcelona approved the protocol.

\section{Outcomes}

Patients' demographic data and clinical assessment of AR were collected during a single visit: type of AR (seasonal, perennial, occupational) and ARIA classification (intermittent or persistent and the severity using the impairment of the four items proposed). The T4SS was also assessed by the total (0 to 12) of nasal symptoms: nasal congestion, rhinorrhoea, sneezing, and nasal itching, scored from 0 (not present) to 3 (intense). In addition, patients were asked to evaluate the global severity of their disease over the last week using a VAS $(0-100 \mathrm{~mm}$ : where 0 is none and 100 represents the maximum severity), and to complete the Rhinitis Quality of Life Questionnaire (RQLQ) to assess AR specific impairment of quality of life. Concomitant diseases and medical treatment received during last month were also collected in the case report form.

\section{Statistical analysis}

The objective of this study was to find the optimal cut-off values of VAS to discriminate between the three categories of AR severity using the already validated ARIA modified criterion classification. Sensitivity, specificity, positive and negative predictive values of each possible cut-off level of VAS from patients with mild, moderate and severe AR were calculated in each case. Decimal values were discarded to facilitate and simplify the use of VAS in clinical practice. The receiver operating characteristic curves (ROC) were calculated to summarize the performance of the VAS classifier across the range of possible VAS scores, using ARIA severity modified classification as the model. In order to evaluate the optimum cut-off level we decided to select the one that had the best sum of the parameters sensibility, specificity, positive and negative predictive values. Correlations between the different severity evaluations were calculated. A non-parametric test was used (Spearman rank correlation) due to a non-normal distribution of the variables. The associations between the categorical variable modified ARIA classification of severity (mild, moderate and severe) with VAS, T4SS and RQLQ were studied using the Cohen's findex.

\section{Results}

From the 5,074 patients originally included in the study, 3,572 patients $(70.4 \%)$ were selected for the reanalysis ( 1,502 being rejected since some of the severity evaluations were not completed). Among them 1,845 patients (51.65\%) were women, the mean age being $37.5 \pm 13.4$ years. 


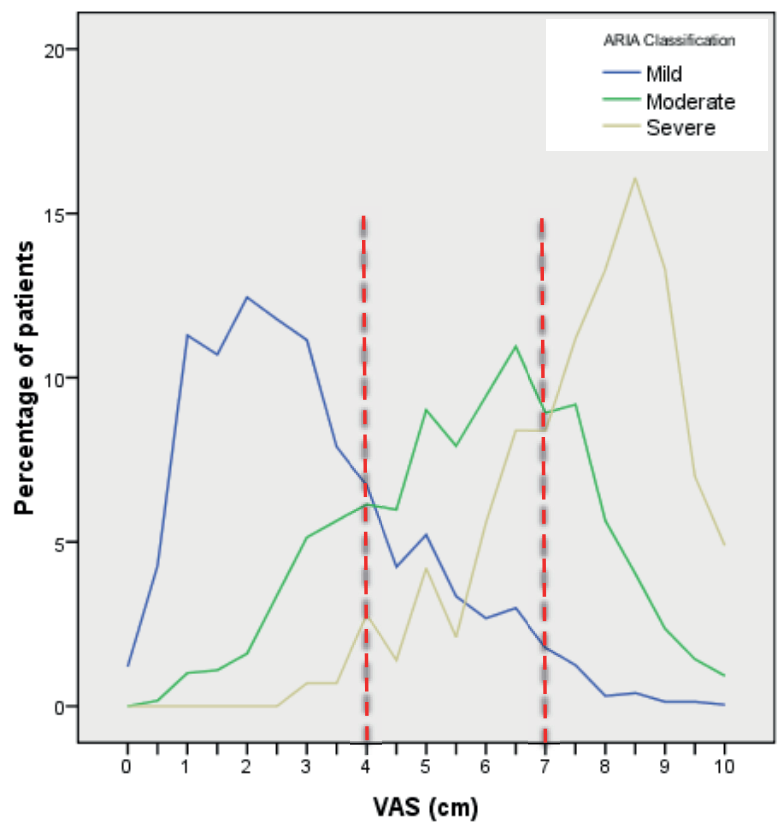

Figure 1. The distribution based on the percentage of patients according to the result of the visual analogue scale for each category of allergic rhinitis severity (mild, moderate and severe). Vertical dashed lines show the best cut-off points for the visual analogue scale to differentiate between mild, moderate and severe allergic rhinitis.

The sample did not show any significant difference in terms of geographical distribution, but moderate and severe AR were significantly more prevalent in patients with persistent $A R$, while mild AR was more prevalent in patients with an intermittent one.

1,280 patients (35.8\%) suffered from mild intermittent AR, 962 (26.9\%) from mild persistent, 515 (14.4\%) moderate intermittent, 672 (18.8\%) moderate persistent, 49 (1.4\%) severe intermittent and 94 (2.6\%) severe persistent. 724 (20.3\%) patients were reported as having concomitant asthma, this being more prevalent in patients with moderate-severe AR $(23.5 \%)$ than with mild AR (18.3\%). All patients included in the study had taken some medication to treat AR during the last month, either continuously or on demand.

VAS showed a significant correlation $(p<0.001)$ with T4SS $(R=0.59)$ and $R Q L Q(R=0.68)$. The modified ARIA classification of severity showed a low association with T4SS $(f=0.44)$, and a moderate association with RQLQ $(f=0.52)$ and VAS $(f=0.62)$. The distribution of patients, according to VAS results, did not differ very much from intermittent to persistent. The VAS medians (interquartile range) resulting for each severity category were: mild intermittent 2.5 (2.5), mild persistent 2.5 (2.5), moderate intermittent 5.7 (3.0), moderate persistent 5.9 (2.7), severe intermittent 7.6 (2.4), severe persistent 7.8 (1.9). Since the severity VAS outcomes between persistent and intermittent were quite similar, the evaluation of symptom duration was discarded when calculating the ROC curves. Table 1 shows the results of sensitivity, specificity, positive and negative predictive values for each possible cut-off level. Figure 1 shows the distribution of patients according to the result of VAS for each AR category of severity.

According to the method selected, the best cut-off values of VAS was 4 to differentiate between mild and moderate and 7 to differentiate between moderate and severe. The area under the ROC curve for each value was 0.85 and 0.90 respectively. Figure 2 shows ROC curves for each cut-off value.

\section{Discussion}

In the present study we have assessed the performance of the VAS as a severity evaluation for AR, using a large number of patients, not only from primary care but also from the specialist setting. The modified ARIA severity classification was used as the model to find the cut-off level, resulting the best cut-off values in 4 and 7 to differentiate between mild, moderate and severe AR. In a previous study by Bousquet et al. ${ }^{(3)}$, the authors concluded that a patient with VAS values under 5 could be classified as mild and over 6 as moderate/severe, obtaining a blind interval for the values between 5 and 6 . In our opinion these results could be related to the heterogeneity of the moderate/severe

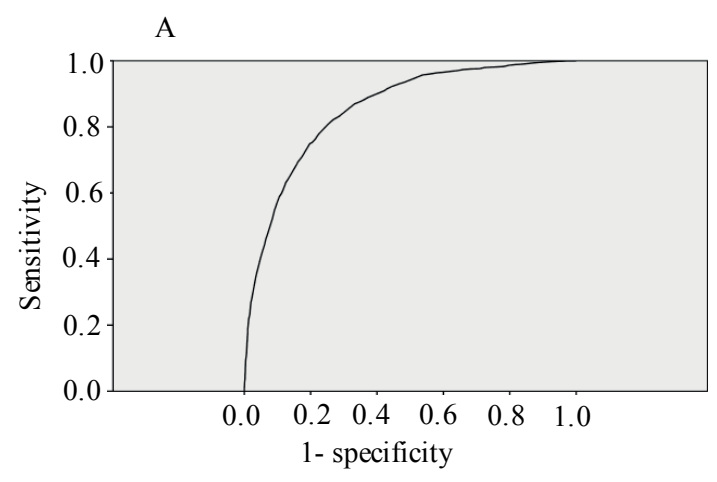

B

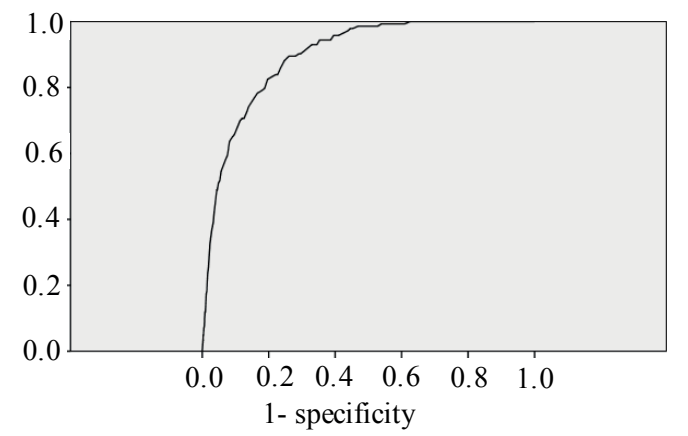

Figure 2. Receiver operating characteristic curves for the best cut-off point of the visual analogue scale (figure 2 A: 4 and figure 2 B: 7 ) to differentiate between mild, moderate and severe allergic rhinitis. 
Table 1. Sensitivity, specificity, as well as positive and negative predictive values of each possible cut off level of the visual analogue scale for mild and severe allergic rhinitis versus the rest in each case.

\begin{tabular}{|c|c|c|c|c|}
\hline \multicolumn{5}{|c|}{ Mild versus the rest } \\
\hline Value & Sensitivity & Specificity & $\begin{array}{l}\text { Positive } \\
\text { predictive } \\
\text { value }\end{array}$ & $\begin{array}{c}\text { Negative } \\
\text { predictive } \\
\text { value }\end{array}$ \\
\hline 2 & 0.971 & 0.356 & 0.472 & 0.953 \\
\hline 3 & 0.909 & 0.579 & 0.562 & 0.915 \\
\hline 4 & 0.808 & 0.747 & 0.655 & 0.868 \\
\hline 5 & 0.685 & 0.843 & 0.721 & 0.819 \\
\hline 6 & 0.519 & 0.917 & 0.787 & 0.763 \\
\hline \multicolumn{5}{|c|}{ Severe versus the rest } \\
\hline Value & Sensitivity & Specificity & $\begin{array}{l}\text { Positive } \\
\text { predictive } \\
\text { value }\end{array}$ & $\begin{array}{c}\text { Negative } \\
\text { predictive } \\
\text { value }\end{array}$ \\
\hline 4 & 0.972 & 0.562 & 0.085 & 0.998 \\
\hline 5 & 0.930 & 0.670 & 0.105 & 0.996 \\
\hline 6 & 0.839 & 0.779 & 0.137 & 0.991 \\
\hline 7 & 0.699 & 0.886 & 0.204 & 0.986 \\
\hline 8 & 0.490 & 0.956 & 0.318 & 0.978 \\
\hline 9 & 0.175 & 0.998 & 0.379 & 0.966 \\
\hline
\end{tabular}

group. We have obtained a different cut-off value to differentiate between mild and the other severity categories of AR than the one obtained in the Bousquet's study (4 versus 5), probably due to the small number of mild patients in that study $(n=195)$, quite different from the patients' distribution in our study (2,242 were mild, 1,187 moderate, and 143 severe).

VAS has been shown to be a valid method of severity assessment in multiple pathologies and primary care physicians are accustomed to its use (9). In chronic rhinosinusitis patients, the VAS has been validated correlating with self-perception of severity and quality of life impairment, resulting in values of 3 and 7 to differentiate between mild, moderate and severe respectively (10).

A recent study has shown that VAS is a simple quantitative tool to assess the burden of AR in primary care, establishing a cut-off variation of $23 \mathrm{~mm}$ of VAS associated with a cut-off variation of 0.5 for the RQLQ. The authors concluded that VAS could detect with high sensitivity the variations of symptoms and quality of life in patients with $A R^{(11)}$.

We have shown in our study that the modified criterion from ARIA guideline to classify AR severity produces categories that are related to the severity of symptoms and the impairment of quality of life, and that VAS can predict these categories of severity. It has been shown previously that VAS scores are worse in patients with more severe disease in terms of symptom score or quality of life ${ }^{(12)}$.

The VAS has also been proposed as a simple clinical control tool for AR in a state of the art document, which defines the concept of severe chronic upper airway disease (SCUAD) as those patients who report bothersome symptoms despite adequate treatment ${ }^{(13)}$. Based on a retrospective study from Bousquet et al. (14) patients reporting total nasal symptoms VAS of 5 or greater, after an adequate treatment for AR, were defined as "uncontrolled". In a consensus document ${ }^{(13)}$ Hellings et al. stressed that VAS validation as a clinical AR control tool is an unmet need. Martins de Oliveira et al. compared a single nasal symptom (nasal obstruction) VAS with an objective measure of nasal flow, the peak nasal inspiratory flow (PNIF) ${ }^{(15)}$. The results showed a lower PNIF in AR patients compared to controls but with no correlation between VAS and PNIF. There was however a weak inverse correlation between PNIF and symptom's score.

The results obtained in our study have some limitations: the values obtained for sensitivity and specificity are not considered optimal (0.808/0.747 for the cut-off point 4 and $0.699 / 0.886$ for the cut-off point 7) but it should be kept in mind that VAS is not a diagnosis test, otherwise it is a tool for assessing severity. Another limitation is that we did not assess the test-retest validity of the VAS nor the changes in severity after treatment. In a study in which VAS was used to assess changes in global evaluation of rhinitis after treatment, the authors concluded that this method is highly responsive to change during the treatment ${ }^{(16)}$.

The VAS scores in patients with moderate to severe AR are similar in Spain ${ }^{(8)}$, France ${ }^{(3)}$ or China ${ }^{(17)}$ so that this simple method can be universally used and compared.

\section{Conclusion}

VAS is a simple, fast and efficient way to assess the severity of $A R$ and can be the ideal tool to use in primary care settings. This study validates the VAS cut-off points of 4 and 7 to differentiate between three severity categories mild, moderate, and severe $A R$, according to the modified ARIA criterion.

\section{Acknowledgements}

Marta Ferrer y Joan Bartra belong to the Red de Investigación de Reacciones Adversas a Alérgenos y Fármacos (RIRAAF) RD 12/0013 of the Instituto de Salud Carlos III. The authors want to thank Lynne Greghorn for the style and language supervision.

\section{Authorship contribution}

ADC developed the project and wrote up the manuscript. VS advised in the methodology and revised the manuscript. JM, JB, ID, MF, IJ, JS, JM and AV revised and corrected the manuscript.

\section{Conflict of interest}

Authors declare no conflict of interest regarding the content of this manuscript. 


\section{References}

1. 1. Bousquet J, Khaltaev N, Cruz AA et al. Allergic Rhinitis and its Impact on Asthma (ARIA) 2008 update (in collaboration with the World Health Organization, GA(2)LEN and AllerGen). Allergy 2008;63 (Suppl 86):8160.

2. 2. Costa DJ, Bousquet PJ, Ryan D et al. Guidelines for allergic rhinitis need to be used in primary care. Prim Care Respir J 2009;18:250-257.

3. 3. Bousquet PJ, Combescure C, Neukirch $F$ et al. Visual analog scales can assess the severity of rhinitis graded according to ARIA guidelines. Allergy 2007;62:367-372.

4. 4. Valero A, Ferrer M, Sastre J et al. A new criterion by which to discriminate between patients with moderate allergic rhinitis and patients with severe allergic rhinitis based on the Allergic Rhinitis and its Impact on Asthma severity items. J Allergy Clin Immunol 2007;120:359-365.

5. 5. Valero A, Ferrer $M$, Baró $E$ et al. Discrimination between moderate and severe disease may be used in patients with either treated or untreated allergic rhinitis. Allergy 2010;65:1609-1613.

6. 6. Montoro J, Del Cuvillo A, Mullol J et al. Validation of the modified allergic rhinitis and its impact on asthma (ARIA) severity classification in allergic rhinitis children: the PEDRIAL study. Allergy 2012;67:1437-1442.

7. 7. Mullol J, Bartra J, del Cuvillo A, Izquierdo I, Muñoz-Cano R, Valero A. Specialist-based treatment reduces the severity of allergic rhinitis. Clin Exp Allergy 2013;43:723-729.
8. 8. del Cuvillo A, Montoro J, Bartra J et al. Validation of ARIA duration and severity classifications in Spanish allergic rhinitis patients - The ADRIAL cohort study. Rhinology 2010;48:201-205.

9. 9. Miller MD, Ferris DG. Measurement of subjective phenomena in primary care research: the Visual Analogue Scale. Fam Pract Res J 1993;13:15-24.

10. 10. Lim M, Lew-Gor S, Darby Y, Brookes $N$, Scadding G, Lund VJ. The relationship between subjective assessment instruments in chronic rhinosinusitis. Rhinology 2007:45:144-147.

11. 11. Demoly P, Bousquet PJ, Mesbah K, Bousquet J, Devillier P. Visual analogue scale in patients treated for allergic rhinitis: an observational prospective study in primary care: asthma and rhinitis. Clin Exp Allergy 2013;43:881-888.

12. 12. Bousquet PJ, Demoly $P$, Devillier $P$, Mesbah K, Bousquet J. Impact of allergic rhinitis symptoms on quality of life in primary care. Int Arch Allergy Immunol 2013;160:393-400.

13. 13. Hellings PW, Fokkens WJ, Akdis C et al. Uncontrolled allergic rhinitis and chronic rhinosinusitis: where do we stand today? Allergy 2013; 68: 1-7.

14. 14. Bousquet PJ, Bachert C, Canonica GW et al. Uncontrolled allergic rhinitis during treatment and its impact on quality of life: a cluster randomized trial. J Allergy Clin Immunol 2010;126:666-668 e661-665.

15. 15. Martins de Oliveira GM, Rizzo JA, Camargos PA, Sarinho ES. Are measure- ments of peak nasal flow useful for evaluating nasal obstruction in patients with allergic rhinitis?. Rhinology 2015;53:160-166.

16. 16. Bousquet P-J, Combescure C, Klossek $J$-M, Daurès J-P, Bousquet J. Change in visual analog scale score in a pragmatic randomized cluster trial of allergic rhinitis. J Allergy Clin Immunol 2009;123:1349-1354.

17. 17. Wang Y, Zhu R, Liu G et al. Prevalence of uncontrolled allergic rhinitis in Wuhan, China: a prospective cohort study. Am J Rhinol Allergy 2014;28:397-403.

\author{
Dr. Alfonso del Cuvillo \\ Rhinitis \& asthma unit \\ UGC ORL, Hospital de Jerez \\ Cádiz \\ Spain \\ Postal Address: Secretaria ORL \\ Hospital de Especialidades de Jerez \\ Carretera de circunvalacion $\mathrm{s} / \mathrm{n}$ \\ Jerez \\ España
}

Fax: +34-956-032302

E-mail:dr.cuvillo@comcadiz.es

ADVERTISEMENT

14th INTERNATIONAL COURSE IN ADVANCED SINUS SURGERY TECHNIQUES

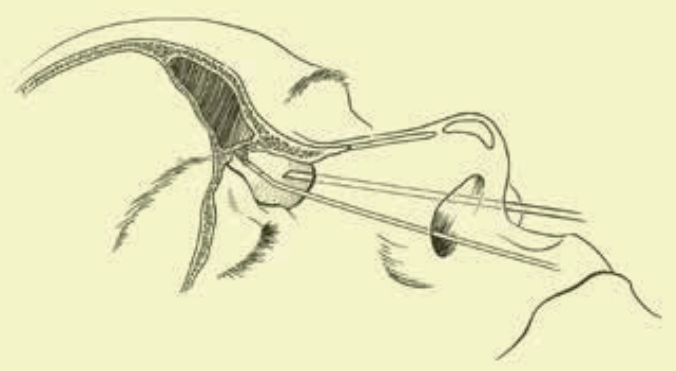

October 23-24-25, 2017

Department of Otorhinolaryngology Academic Medical Center of the University of Amsterdam The Netherlands

For further information contact Wytske J. Fokkens, MD, PbD ENT dept. AMC Course Secretariat

Tel: 0031205668586 / Fax 0031205669573

Email:m.b.vanhuiden@amc.uva.nl Web: wnw.sinuscourse.nl 